\title{
Correlation of reduced amniotic fluid index with neonatal outcome
}

\author{
Rachna Chaudhary*, Vandana Dhama, Shakun Singh, Madhumita Singh
}

Department of Obstetrics and Gynecology, LLRM Medical College, Meerut, Uttar Pradesh, India

Received: 16 March 2017

Accepted: 21 April 2017

\section{*Correspondence:}

Dr. Rachna Chaudhary,

E-mail: drrachnachaudhary@gmail.com

Copyright: (c) the author(s), publisher and licensee Medip Academy. This is an open-access article distributed under the terms of the Creative Commons Attribution Non-Commercial License, which permits unrestricted non-commercial use, distribution, and reproduction in any medium, provided the original work is properly cited.

\begin{abstract}
Background: Amniotic fluid assessment is an essential part of evaluation of fetus health in terms of fetal distress, meconium aspiration, IUGR, Non-reassuring fetal heart rate patterns, birth asphyxia, low birth weight, low Apgarscores and NICU Admissions.

Methods: This prospective observational study included 200 antenatal patients of term gestation of which 100 cases with $\mathrm{AFI}<8$ and 100 controls with $\mathrm{AFI} \geq 8$ but $\leq 15$ were included in study. Ultrasonography and NST were done and results of both groups compared for the presence of meconium passage, non-reactive NST, birth asphyxia, low Apgarscores, low birth weight, NICU admissions and neonatal deaths.

Results: There was increased incidence of non-reactive NST (40\% vs 12\%), meconium stained (33\% vs 10\%) in oligohydramnios group. In cases $49 \%$ of patients had vaginal delivery while in controls $78 \% .51 \%$ underwent LSCS in cases while in controls 22\%. Apgar score <7 was found in 7\% vs $4 \%$. Birth weight was $<2.5 \mathrm{~kg}$ in $35 \%$ vs $13 \%$. NICU admissions in study group was $15 \%$ vs $11 \%$. The neonatal death was similar in both cases groups.

Conclusions: Oligohydramnios affect the neonatal outcome in terms of meconium stained liquor, non-reassuring fetal heart rate, low birth weight, birth asphyxia, low Apgar-scores and NICU Admissions and neonatal death but the difference was not statistically significant. Early detection of oligohydramnios and its management may help in reduction of perinatal morbidity and decreased caesarean deliveries.
\end{abstract}

Keywords: Cesarean delivery, Neonatal outcome, Non-stress test, Oligohydramnios

\section{INTRODUCTION}

Modern obstetrics is concerned with the health and wellbeing of both the mother and the unborn child. Amniotic fluid volume is a variable that has got great impact on the fetal condition in the intrapartum period. Clinical estimation of amniotic fluid volume is an important part of fetal assessment as variation in its amount has been related to a variety of pregnancy complications. $^{1,2}$ Amniotic fluid assessment is an essential part of evaluation of maternal and fetal health in terms of fetal distress, meconium aspiration, termination of pregnancy by caesarean section, Intra uterine growth restriction (IUGR), non-reassuring fetal heart rate patterns, birth asphyxia, low Apgar-scores at birth and neonatal intensive care unit admissions. ${ }^{3}$ It is also associated with maternal morbidity in the form of congenital malformations, increased rate of induction or operative interference. Early detection of oligohydramnios and its management may help in reduction of perinatal morbidity and mortality on one side and decreased caesarean deliveries on the other side. Since oligohydramnios has got significant impact on neonatal outcome and maternal morbidity, it prompted us to do this study.

Amniotic fluid index, as described by Phelan and coworkers, in 1987, remains one of the most commonly used methods of amniotic fluid volume assessment. In this method, the ultrasound transducer is held perpendicular to the floor and parallel to the long axis of the pregnant women. The uterus is divided into four equal 
quadrants - the right and left upper and lower quadrants, respectively. The AFI is the sum of the single deepest pocket from each quadrant. A fluid pocket may contain fetal parts or umbilical cord loops but these are not included in the measurement. ${ }^{4}$ The normal range for AFI that is most commonly used is 5 to $24 \mathrm{~cm}$, with values above and below this indicating hydramnios and oligohydramnios respectively. ${ }^{5}$

Oligohydramnios is defined as an amniotic fluid index below the $5^{\text {th }}$ centile for the gestational age as per Moore and Cayle. ${ }^{6}$ The sonographic diagnosis of oligohydramnios is usually based on an AFI $\leq 5 \mathrm{~cm}$ or on a single deepest pocket of amnionic fluid $\leq 2 \mathrm{~cm}$. ${ }^{7}$ Phelan defined oligohydramnios as amniotic fluid index (AFI) $\leq 5 \mathrm{~cm}$ and borderline oligohydramnios as AFI between 5 and $8 \mathrm{~cm}$ between $36-42$ weeks of gestation. ${ }^{4}$ It complicates approximately 1 to 5 percent of pregnancies at term.

The aim of our study was to determine the correlation of reduced amniotic fluid index (AFI) with neonatal outcome in terms of - meconium stained liquor, nonstress test (NST), period of gestation at birth, mode of delivery (spontaneous or induced normal vaginal delivery, elective or emergency LSCS), Weight of baby at birth, Apgar score at birth, admission to NICU, neonatal deaths, correlation of neonatal outcome with severity of oligohydramnios.

\section{METHODS}

The study was conducted in the department of Obstetrics and Gynaecology, LLRM Medical College, Meerut. This was a prospective observational study carried out for sixteen months from June 2015 to September 2016. 200 cases of antenatal women who attended or got admitted, out of which 100 cases with $\mathrm{AFI} \leq 8$ (oligohydramnios) as study group and 100 cases with AFI $>8$ but $\leq 15$ as control group as per inclusion and exclusion criteria.

\section{Inclusion criteria}

- Singleton pregnancy

- Gestational age between 37-42 weeks

- Intact membranes at the time of antepartum testing

- AFI assessment within seven days of delivery or at onset of labor

- Delivering at study institution

- No gross congenital anomaly.

\section{Exclusion criteria}

- Multiple pregnancy

- Malpresentations i.e breech or transverse lie

- Premature rupture of membranes

- Antepartum haemorrhage

- Pregnancy with fetal death (IUD)
- High risk pregnancy (Hypertension, Diabetes, Severe Anemia)

- Pregnancy with absolute indication for LSCS.

A careful clinical history was taken from all the patients particularly about age, last menstrual period, history of previous menstrual cycles, history of oral contraceptive use before the last menstrual period, previous obstetric history and obstetric complications.

A thorough clinical examination including blood pressure, presence of pedal edema, pallor was examined. By obstetric palpation gestational age, presentation and amount of liquor was noted. The fetal heart rate was monitored with auscultation and cardiotocography. All preliminary and baseline investigations like hemoglobin estimation, blood grouping and typing and complete urine examination was done. Then patients fulfilling all the inclusion and exclusion criteria were included in the study.

With all the criteria satisfied, all subjects were evaluated in the department of Radiodiagnosis for four-quadrant AFI measurements, as described by Phelan as well as gestational age assessment, fetal presentation, placental localization and to rule out any gross congenital anomaly in the fetus. The gestational age was assessed by standard fetal biometry and those between 37 to 42 weeks of gestation were considered for study. The AFI is the sum of the single vertical deepest pocket from each quadrant. A fluid pocket may contain fetal parts or umbilical cord loops but these are not included in the measurement. ${ }^{4}$ Patients having gross congenital malformations and intrauterine fetal death were excluded from the study.

According to the measurement of AFI, two groups were formed as:

- Cases $\mathrm{AFI} \leq 8 \mathrm{~cm}$

- Control AFI $>8 \mathrm{~cm}$ but $\leq 15 \mathrm{~cm}$

On admission to the labour room an admission NST was done as per WHO protocols. An NST was considered reactive when the baseline fetal heart rate was between 110 and 160 beats per minute and when there were at least two FHR acceleration of 15 beats per minute lasting at least 15 seconds were present within a 20 minute period. An abnormal fetal heart rate tracing was defined as the presence of severe variable or late decelerations or a bradycardia. The diagnosis of fetal distress was based on repetitive decelerations or prolonged bradycardia or meconium staining of liquor. Those with suspicious NST were reassessed after change in maternal position and adequate hydration and included among reactive or nonreactive group. Labour was either spontaneous or induced in both study and control group. During labour intermittent auscultation of fetal heart rate was done to detect any signs of fetal distress. The nature of amniotic fluid was noted at the time of rupture of membrane and was classified as clear, thin meconium stained liquor and 
thick meconium stained liquor. Progress of labour was monitored on a partogram.

Mode of delivery (vaginal, elective LSCS, or emergency LSCS) and labour management were at the decision of obstetrician in charge. After delivery, the baby was handed over to the attending pediatrician who was unaware of the ultrasound findings.

Records were reviewed for the presence of non-reactive NST, abnormal fetal heart rate tracing, induction of labour, mode of delivery, meconium passage, birth weight, Apgar score, neonatal intensive care unit admissions and neonatal deaths.

\section{Statistical analysis}

Data were compared and analyzed. The results are presented as mean $\pm \mathrm{SD}$. The Chi-square test was used to compare the categorical variables. The $p$-value $<0.05$ was considered significant. All the analysis was carried out on SPSS 16.0 version (Chicago, Inc., USA).

\section{RESULTS}

The patients in both study and control group in our study were comparable in terms of different demographic factors like age, parity, booking status, socioeconomic status, residential background and also period of gestation.

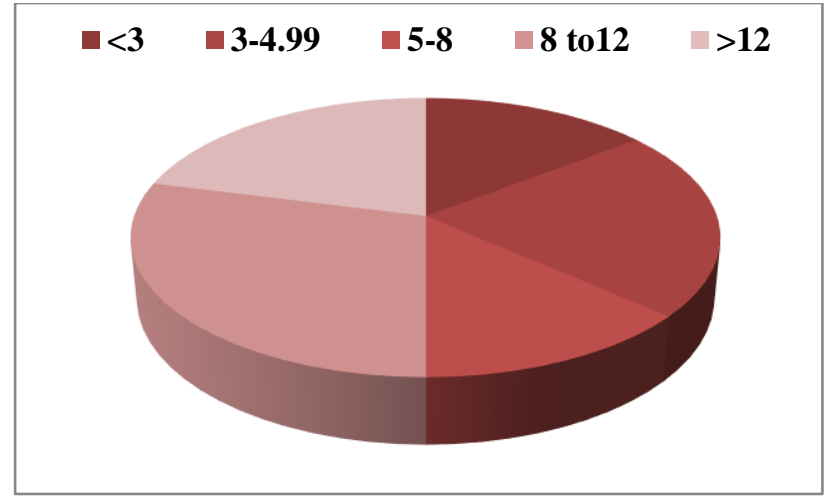

Figure 1: Distribution of AFI in study group and control group.

Figure 1 shows the distribution of AFI in study group and control group. AFI was moderate $(3-4.99 \mathrm{~cm})$ in more than one third of the cases (44\%). Severe oligohydramnios (AFI $<3 \mathrm{~cm}$ ) was present in $29 \%$ of patients and mild oligohyramnios (AFI $5-8 \mathrm{~cm}$ ) in $27 \%$ of patients. AFI was $>12 \mathrm{~cm}$ in more than one third of the controls (42\%) and 58\% had AFI $8-12 \mathrm{~cm}$.

$40 \%$ of the patients in cases had non-reactive NST while in controls only $12 \%$ had non-reactive NST. Meconium stained liquor was in $33 \%$ in cases while in $10 \%$ among control group.

Table 1: NST pattern and colour of amniotic fluid in study and control group.

\begin{tabular}{|c|c|c|c|c|c|}
\hline \multirow{2}{*}{ NST pattern } & \multicolumn{2}{|c|}{ Study group $(\mathrm{n}=100)$} & \multicolumn{2}{|c|}{ Control group $(n=100)$} & \multirow{2}{*}{ OR $(95 \%$ CI $)$, p-value ${ }^{1}$} \\
\hline & No. & $\%$ & No. & $\%$ & \\
\hline Reactive & 60 & 60.0 & 88 & 88.0 & $0.20(0.09-0.42), 0.0001 *$ \\
\hline Non-reactive & 40 & 40.0 & 12 & 12.0 & 1.00 (Ref.) \\
\hline Colour of amniotic fluid & $\mathrm{n}=10 \mathrm{c}$ & & $\mathrm{n}=1 \mathrm{c}$ & & \\
\hline Clear & 67 & 67.0 & 90 & 90.0 & 1.00 (Ref.) \\
\hline Thin MSL & 20 & 20.0 & 5 & 5.0 & $5.37(1.91-15.04), 0.001 *$ \\
\hline Thick MSL & 13 & 13.0 & 5 & 5.0 & $3.49(1.18-10.27), 0.02 *$ \\
\hline
\end{tabular}

Table-2: Comparison of LSCS for fetal distress with NST in study and control group

\begin{tabular}{|c|c|c|c|c|c|}
\hline \multirow{2}{*}{ NST } & \multicolumn{2}{|c|}{ Study group $(n=26)$} & \multicolumn{2}{|c|}{ Control group $(n=16)$} & \multirow{2}{*}{ OR $(95 \%$ CI $)$ p-value ${ }^{1}$} \\
\hline & No. & $\%$ & No. & $\%$ & \\
\hline Reactive & 1 & 3.8 & 7 & 43.8 & $0.05(0.01-0.47), 0.001 *$ \\
\hline Non-reactive & 25 & 96.2 & 9 & 56.2 & 1.00 (Ref.) \\
\hline
\end{tabular}

LSCS for fetal distress in non-reactive NST was $96.2 \%$ in cases and $56.2 \%$ in controls. Table 3 shows the comparison of NST pattern with perinatal outcome in study group. LSCS for fetal distress was in $20 \%$ in patients with reactive NST and $89.3 \%$ in patients with non-reactive NST. Clear liquor was in $80 \%$ of reactive and in $45 \%$ of non-reactive NST group and the difference was significant $(\mathrm{p}=0.0004)$. APGAR score $<7$ was significantly $(\mathrm{p}=0.02)$ lower in reactive $(1.6 \%)$ than nonreactive $(12.5 \%)$. The NICU admissions was found to be insignificantly $(\mathrm{p}>0.05)$ lower in reactive NST $(9.8 \%)$ than non-reactive NST $(22.5 \%)$. There was no neonatal death among reactive NST group while 1 neonatal death $(2.5 \%)$ was found in non-reactive group. 
Table 3: Comparison of NST with perinatal outcome in study group.

\begin{tabular}{|c|c|c|c|c|c|}
\hline \multirow{2}{*}{$\begin{array}{l}\text { Perinatal } \\
\text { Outcome }\end{array}$} & \multicolumn{2}{|c|}{$\begin{array}{l}\text { Reactive } \\
\mathrm{n}=60\end{array}$} & \multicolumn{2}{|c|}{$\begin{array}{l}\text { Non-reactive } \\
n=40\end{array}$} & \multirow{2}{*}{$\begin{array}{l}\text { p- } \\
\text { value }^{1}\end{array}$} \\
\hline & No. & $\%$ & No. & $\%$ & \\
\hline Fetal distress & 1 & 20.0 & 25 & 89.3 & - \\
\hline \multicolumn{6}{|l|}{ Liquor } \\
\hline Clear & 49 & 80.3 & 18 & 45.0 & \multirow[t]{3}{*}{$0.0004 *$} \\
\hline Thin MSL & 8 & 13.1 & 12 & 30.0 & \\
\hline Thick MSL & 3 & 4.9 & 10 & 25.0 & \\
\hline \multicolumn{6}{|c|}{ APGAR score (at $5 \mathrm{~min}$. of birth) } \\
\hline$<7$ & 1 & 1.6 & 5 & 12.5 & \multirow{2}{*}{$0.025^{*}$} \\
\hline$\geq 7$ & 59 & 98.3 & 35 & 87.5 & \\
\hline \multicolumn{6}{|c|}{ NICU admissions } \\
\hline Yes & 6 & 9.83 & 9 & 22.5 & \multirow{2}{*}{$0.08-$} \\
\hline No & 54 & 90.1 & 31 & 77.5 & \\
\hline \multicolumn{6}{|c|}{ Neonatal death } \\
\hline Yes & 0 & 0.0 & 1 & 2.5 & \multirow{2}{*}{$0.21-$} \\
\hline No & 60 & 100.0 & 39 & 97.5 & \\
\hline
\end{tabular}

Table 4 shows the comparison of perinatal outcome with MSL in study group. Perinatal outcome was comparatively poor in those with meconium stained liquor in terms of low Apgar score, more neonatal admissions and neonatal deaths.

Table 4: Comparison of perinatal outcome with MSL in study group.

\begin{tabular}{|lll|}
\hline Perinatal outcome & \multicolumn{2}{l|}{ Study group $(\mathbf{n = 3 3})$} \\
\hline NICU Admissions & No. & $\%$ \\
\hline Apgar score $<7$ & 12 & 36.3 \\
\hline Neonatal death & 6 & 18.1 \\
\hline
\end{tabular}

The mean Apgar score in study group is 8.37 while in controls it is 8.34. Apgar score $<7$ was found in $7 \%$ of cases and $4 \%$ of controls. APGAR score $<7$ was insignificantly $(\mathrm{p}>0.05)$ higher in study group than control group. Mean birth weight in study group is 2.65 $\mathrm{kg}$ and $2.77 \mathrm{~kg}$ in controls. Birth weight was $<2.5 \mathrm{~kg}$ in $35 \%$ of cases and $13 \%$ of controls. Birth weight $<2.5 \mathrm{~kg}$ was significantly $(\mathrm{p}=0.0001)$ higher in study group than control group.

Table-5: Comparison of perinatal outcome in study and control group.

\begin{tabular}{|c|c|c|c|c|c|}
\hline \multirow{2}{*}{ Perinatal outcome } & \multicolumn{2}{|c|}{ Study group $(n=100)$} & \multicolumn{2}{|c|}{ Control group $(\mathrm{n}=\mathbf{1 0 0})$} & \multirow{2}{*}{ OR $(95 \%$ CI $)$, p-value ${ }^{1}$} \\
\hline & No. & $\%$ & No. & $\%$ & \\
\hline \multicolumn{6}{|c|}{ APGAR score (at 5 min. of birth) } \\
\hline$<7$ & 7 & 7.0 & 4 & 4.0 & $1.80(0.51-6.37), 0.35$ \\
\hline$\geq 7$ & 93 & 93.0 & 96 & 96.0 & 1.00 (Ref.) \\
\hline Mean \pm SD & \multicolumn{2}{|c|}{$8.37 \pm 0.96$} & \multicolumn{2}{|c|}{$8.34 \pm 0.86$} & \\
\hline \multicolumn{6}{|l|}{ Birth weight in $\mathrm{kg}$} \\
\hline$<2.5$ & 35 & 35.0 & 13 & 13.0 & $3.60(1.76-7.35), 0.0001 *$ \\
\hline$\geq 2.5$ & 65 & 65.0 & 87 & 87.0 & 1.00 (Ref.) \\
\hline Mean \pm SD & \multicolumn{2}{|c|}{$2.65 \pm 0.40$} & \multicolumn{2}{|c|}{$2.77 \pm 0.32$} & \\
\hline \multicolumn{6}{|l|}{ Admission in NICU } \\
\hline Yes & 15 & 15.0 & 11 & 11.0 & $1.42(0.62-3.28), 0.40$ \\
\hline No & 85 & 85.0 & 89 & 89.0 & 1.00 (Ref.) \\
\hline \multicolumn{6}{|l|}{ Neonatal deaths } \\
\hline Yes & 1 & 1.0 & 1 & 1.0 & $1.00(0.06-16.21), 1.00$ \\
\hline No & 99 & 99.0 & 99 & 99.0 & 1.00 (Ref.) \\
\hline
\end{tabular}

NICU admissions in study group was $15 \%$ in cases and $11 \%$ in controls. NICU admission was insignificantly $(\mathrm{p}>0.5)$ higher in study group than control group. The neonatal death was similar in both cases and controls.

There was 1 neonatal death in both the groups and both were due to meconium aspiration. Average duration of stay in NICU in study group was 8.21 days and 5.33 days in control group.

NICU stay was insignificantly $(p>0.5)$ higher in study group than control group. Perinatal outcome worsens as the severity of oligohydramnios increases but the difference is not statistically significant.

Table 6: Comparison of hospital stay of NICU admissions between cases and controls.

\begin{tabular}{|ll|} 
Groups & $\begin{array}{l}\text { Hospital stay in days } \\
(\text { Mean } \pm \text { SD) }\end{array}$ \\
\hline Cases & $8.21 \pm 4.88$ \\
\hline Controls & $5.33 \pm 3.04$ \\
\hline p-value $^{1}$ & 0.13 \\
\hline${ }^{1}$ Unpaired t-test & \\
\hline
\end{tabular}


Table 7: Comparison of AFI with perinatal outcome in study group.

\begin{tabular}{|c|c|c|c|c|c|c|c|}
\hline \multirow{2}{*}{ Perinatal Outcome } & \multicolumn{2}{|c|}{$<3(n=29)$} & \multicolumn{2}{|c|}{$3-4.99(n=44)$} & \multicolumn{2}{|c|}{$5-8(n=27)$} & \multirow{2}{*}{ p-value ${ }^{1}$} \\
\hline & No. & $\%$ & No. & $\%$ & No. & $\%$ & \\
\hline \multicolumn{8}{|l|}{ Birth weight in kg } \\
\hline$<2.5$ & 15 & 51.7 & 12 & 27.3 & 8 & 29.6 & \multirow{2}{*}{0.08} \\
\hline$\geq 2.5$ & 14 & 48.3 & 32 & 72.7 & 19 & 70.4 & \\
\hline \multicolumn{8}{|l|}{ APGAR score } \\
\hline$<7$ & 2 & 6.9 & 5 & 11.4 & 0 & 0.0 & \multirow{2}{*}{0.19} \\
\hline$\geq 7$ & 27 & 93.1 & 39 & 88.6 & 27 & 100.0 & \\
\hline \multicolumn{8}{|l|}{ NICU admissions } \\
\hline Yes & 4 & 13.8 & 10 & 22.7 & 1 & 3.7 & \multirow{2}{*}{0.02} \\
\hline No & 25 & 86.2 & 34 & 77.3 & 26 & 96.3 & \\
\hline \multicolumn{8}{|l|}{ Neonatal death } \\
\hline Yes & 0 & 0.0 & 1 & 2.3 & 0 & 0.0 & \multirow{2}{*}{0.52} \\
\hline No & 29 & 100.0 & 43 & 97.7 & 27 & 100.0 & \\
\hline
\end{tabular}

Only NICU admission was significantly ( $\mathrm{p}=0.02)$ associated with AFI. There was only1 neonatal death and it was in those with moderate oligohydramnios associated with thick meconium aspiration.

\section{DISCUSSION}

The patients in both study and control group in our study were comparable in terms of different demographic factors like age, parity, booking status, socioeconomic status, residential background and also period of gestation. Comorbid conditions like hypertension, diabetes, severe anemia, antepartum haemorrhage, malpresentations had been excluded from both the groups.

The rate of non-reactive NST is $40 \%$ in study group and $12 \%$ in control group in present study and is comparable to results in similar studies as in study by Patel PK et al, Padmini CP et al, Kaur P et al and Kumar P et al it was $65 \%, 40 \%, 38 \%$, and $40 \%$ respectively. ${ }^{8,9,11,16}$

The occurrence of meconium stained amniotic fluid is high in women with oligohydramnios. The meconium stained liquor was noted in $33 \%$ in study group while $10 \%$ in control group which is statistically significant and consistent with the studies done before as in Kaur P et al, Padmini CP et al, Patel PK et al meconium stained liquor was found in $48 \%, 48 \%$ and $51 \%$ respectively. ${ }^{9,8,11}$

In the present study, among cases 49 out of the 100 patients $(49 \%)$ had vaginal delivery and 51 patients (51\%) underwent lower segment caesarean section. While in controls 78 out of 100 patients $(78 \%)$ had normal vaginal delivery and $22(22 \%)$ underwent lower segment cesarean section Thus, there was increased risk of obstetric interventions in patients with oligohydramnios in the form of LSCS and was similar to results in various studies like in Padmini CP et al, Kumar $\mathrm{P}$ et al, Biradar KD et al, Moses $\mathrm{V}$ et al rate of cesarean section was 62\%, 63\%, $62 \%$ and $42 \%$ respectively. $8,16,10,12$

The mean birth weight was low in oligohydramnios group. The percentage of low birth weight in oligohydramnios group was $35 \%$ while in control group was $13 \%$ which is statistically significant. The occurrence of low birth weight was $35 \%$ which is comparable with that of other studies as Bhagat et al, Biradar KD et al in which the percentage of low birth rate was $56 \%$ and $38.6 \%$ respectively. ${ }^{7,10}$

Apgar score $<7$ at 5 minutes of birth was found in $7 \%$ patients in those with oligohydramnios and $4 \%$ in normal AFI group which is not statistically significant. The results are comparable with that of other studies such as in Umber et al, Padmini CP et al, Bhagat Chawla et al the percentage of babies with Apgar score $<7$ at 5 minutes of birth was $6 \%, 8 \%$ and $4 \%$ respectively. $8,13,7$

Admissions to neonatal intensive care unit were similar in both oligohydramnios and those with normal AFI. NICU admissions were $15 \%$ in oligohydramnios group due to various morbidities like birth asphyxia and meconium aspiration. This result is comparable to result of studies like Umber et al, Casey et al and Patel PK et al in which neonatal admissions were $7 \%, 7 \%$, and $20 \%$ respectively. ${ }^{13,14,11}$

This result was not comparable to result of various studies in which neonatal admissions in those with oligohydramnios were high like Kaur $\mathrm{P}$ et al, Chandra $\mathrm{P}$ et al, Padmini CP et al. ${ }^{9,8,15}$ Neonatal admissions in patients with oligohydramnios in study by Bhagat et al was very high, equal to $92 \%$. Neonatal admissions were less in our study as compared to others as we had excluded associated comorbidities like hypertension, diabetes, severe anemia. ${ }^{7}$ 


\section{CONCLUSION}

Thus, this study suggest that Oligohydramnios affect the neonatal outcome in terms of meconium stained liquor, non-reassuring fetal heart rate, low birth weight, birth asphyxia, low Apgar-scores at birth and Neonatal intensive care unit Admissions and neontal death but the difference is not statistically significant. Regular antenatal and intranatal monitoring should be done with the help of non-stress test and biophysical profile to diagnose any fetal compromise at the earliest. Timely intervention if taken can reduce perinatal morbidity and mortality. Early detection of oligohydramnios and its management may help in reduction of perinatal morbidity and decreased caesarean deliveries. Vaginal delivery and caesarean section should be well balanced so that unnecessary maternal morbidities are prevented and labour outcome improved.

\section{Funding: No funding sources}

Conflict of interest: None declared

Ethical approval: The study was approved by the Institutional Ethics Committee

\section{REFERENCES}

1. Chamberlain PF, Manning FA, Morrison I, et al. The relationship of marginal and decreased amniotic fluid volumes to perinatal outcome. Am J Obstet Gynecol. 1984;150(3):245-49

2. Nageotte MP, Towers CV, Asrat T, Freeman RK. Perinatal outcome with the modified biophysical profile. Am J Obstet Gynecol. 1994;170(6):1672-6.

3. Ali HS. Assessment of amniotic fluid index in normal pregnancy at a tertiary care hospital setting. J Ayub Med Coll Abbottabad. 2009;21:149-51.

4. Phelan JP, Smith CV, Broussard P, Small M. Amniotic fluid volume assessment with the fourquadrant technique at 36-42 weeks' gestation. J Reprod Med. 1987;32:540-2.

5. William's Obstetrics, 24th edition; Cunningham, Leveno, Bloom, Dashe. McGraw Hill;233.

6. Moore TR, Cayle JE. The amniotic fluid index in normal human pregnancy. Am J Obstet Gynecol. 1990;162(5):1168.
7. Bhagat M, Chawla Indu: Correlation of Amniotic Fluid Index with Perinatal outcome, J Obstetr Gynaecol India. 2014;64(1):32-5.

8. Padmini CP, Chaitra R, Indra N, Sriram AM. A prospective study of effect of amniotic fluid index less than 5 at term on perinatal outcome. Int J Reprod Contracept Obstet Gynecol. 2016;5:1732-5

9. Kaur P, Desai DA, Taraiya A. A study on the perinatal outcome in cases of oligohydramnios. Int $\mathbf{J}$ Reprod Contracept Obstet Gynecol. 2016;5:98-109.

10. Biradar KD, Shamanewadi AN. Maternal and perinatal outcome in oligohydramnios: study from a tertiary care hospital, Bangalore, Karnataka, India. Int J Reprod Contracept Obstet Gynecol. 2016;5:2291-4

11. Patel PK, Pitre DS, Gupta H. Pregnancy outcome in isolated oligohydramnios at term. National J Comm Med. 2015;6(2):84-8.

12. Moses V, Thakre S. A study of maternal and fetal outcome in third trimester diagnosed cases of oligohydramnios. IJRCOG. 2016:5:2944-8.

13. Umber A. Perinatal outcome in pregnancies complicated by isolated oligohydramnios at term. Annals. 2009;15:35-7.

14. Casey BM, McIntire DD, Bloom SL, Lucas MJ, Santos R, Twickler DM et al. Pregnancy outcomes after antepartum diagnosis of oligohydramnios at or beyond 34 weeks gestation. Am J Obstet Gynecol. 2000;182(4):909.

15. Chandra P, Kaur SP, Hans DK, Kapila AK. The impact of amniotic fluid assessed intrapartuman perinatal outcome. Obstet Gynaecol Today. 2000;5(8):478-81.

16. Kumar P, Iyer S, Ramkumar V. Amniotic fluid index-a new ultrasound assessment of amniotic fluid. J Obstet Gynecol India. 1991;41(1);10-2.

Cite this article as: Chaudhary R, Dhama V, Singh $\mathrm{S}$, Singh M. Correlation of reduced amniotic fluid index with neonatal outcome. Int J Reprod Contracept Obstet Gynecol 2017;6:2401-6. 